\title{
Robotic Manipulators Inspired by Cephalopod Limbs*
}

\author{
William McMahan, Bryan Jones, Ian Walker, Vilas Chitrakaran, Arjun Seshadri, \\ and Darren Dawson \\ Department of Electrical and Computer Engineering \\ Clemson University \\ Clemson, South Carolina 29634 USA \\ \{wmcmaha, bryanj, iwalker, cvilas, aseshad, ddawson\}@clemson.edu
}

\begin{abstract}
This paper connects the investigation of the biomechanics and behavior of octopus in the performance of a wide range of dexterous manipulations to the creation of octopus arm-like robots. This is achieved via the development of a series of octopus arm models which aid in both explaining the underlying octopus biomechanics and in developing a specification for the design of robotic manipulators. Robotic manipulators which match the key features of these models are then introduced, followed by the development of inverse kinematics for the circular (constant) curvature model.
\end{abstract}

Index Terms - Biological Inspiration, Robotics, Robot Manipulator, Design, Inverse Kinematics

\section{Introduction}

Tongues, trunks, and tentacles demonstrate an amazing variety of abilities by which animals dexterously interact with and manipulate their environment. For instance, giraffes wrap their tongues around high tree branches to strip off leaves. They can also use their tongue to more delicately pick off leaves while avoiding the many spiny thorns found in acacia trees. Elephant trunks are amazing appendages that are strong enough to knock over trees and yet agile enough to pick straw off the ground. Squids have ten arms, two of which are tentacles. These tentacles are specialized arms used specifically for quick-strike prey capture. The other arms on the squid, which are similar to octopus arms, allow the creature to perform more general prey/object manipulation and locomotion.
These biological manipulators serve as a source of inspiration for the design and application of robotic manipulators [1]. For instance, a search-and-rescue robot equipped with "octopus arms" could efficiently navigate a rubble pile, searching for victims in crevices or analyzing structural integrity in a collapsed building. The variable stiffness observed in an elephant's trunk would allow a similar robot to grasp and handle payloads of varying dimensions and fragility (e.g. rocks and humans). Manipulators designed with redundant degrees of freedom can navigate around obstacles while performing end-effector tasks just as the giraffe tongue can.

However, it is no small task to create robotic manipulators that replicate the abilities of biological manipulators. Thus far, researchers have designed and built a number of robots that try to duplicate the behavior exhibited by tongues ([2]), trunks ([3], [4], [5], [6], [7]), and tentacles ([8], [9], [10], [11]) to varying degrees of success.

It is important to note that it is rarely practical (or desirable) to build robotic devices with "biological designs". In many cases, present technology does not possess the materials and actuators to allow for it. Biological systems are composed of biological components, which are quite different from the components available for robotics systems. Additionally, evolution (the biological design process) is less concerned with optimal performance than with merely performing well enough to contend successfully within its economic niche. Evolution also has the constraint of being limited to modifying of an existing system, which may create innovative, but sub-optimal designs ([12], [13, p. 20-38]).

\footnotetext{
* This work was supported in part by the Defense Advanced Research Projects Agency (DARPA) Defense Sciences through the Space and Naval Warfare Systems Center, San Diego, Contract Number N66001-03-C-8043.
} 
Instead, the value of biology to engineering (and engineering to biologists) lies in creative inspiration and the practice of modeling systems. For instance, a model of the musculature provides biological insight into the operation of the creature by showing the complex relationship between muscle forces and the resulting deformation and movement of the arm. The insight from this complex relationship can also provide information as to control strategies and actuator arrangement. In addition, the assumptions embedded in these models define the type of mechanism necessary to replicate the actions produced by the model. A number of models that allow kinematic or dynamic analysis of tongue, trunk, and tentacle-like robots have been proposed. These include [14], [15], [4], [16], and [17].

This paper discusses biological investigation of the structure and behavior of the octopus and relates it to the design of robotic manipulators which possess properties similar to those of an octopus arm, through the use of models. The work is being conducted as part of the multidisciplinary and multi-institutional Soft Robot Manipulators and Manipulation project currently being funded by the DARPA BIODYNOTICS Program.

Section 2 provides an overview of fundamental biological research that is taking place under the above project. This research includes investigations on the morphology and biomechanics of octopus arms (being performed at the University of North Carolina, Chapel Hill, NC by Dr. William Kier), and investigations of octopus behavior (conducted at the Marine Biological Laboratory, Woods Hole, MA by Dr. Roger Hanlon).

The project also features basic biological research in the neural control of octopus (conducted at the Hebrew University of Jerusalem by Dr. Binyamin Hochner), and consequent modeling efforts (being performed at the Weizmann Institute, Israel, by Dr. Tamar Flash, and at Brooklyn College, New York, by Dr. Frank Grasso). Section 3 summarizes these models, which are based strongly upon observations obtained from the biological investigations.

A key goal for the project is to use insights from the biological investigations to design and apply biologically inspired soft tentacle robots. The design efforts are led by Penn State University, under Dr. Christopher Rahn and Dr. Qiming Zhang. Section 3 also summarizes some designs for implementing key aspects of the biology-based models in robot hardware.

Given a manipulator design, novel but efficient kinematic and control algorithms together with intuitive operator interfaces, are required for effective deployment. This aspect of the project is led at Clemson Uni- versity by Dr. Ian Walker, Dr. Darren Dawson, and Dr. Christopher Pagano. Sections 4 and 5 focus on one design model in particular and presents novel inverse kinematics which enables the design and construction of a tendon-driven, variable stiffness manipulator.

\section{Octopus Arm Biology}

The amazing range of abilities octopus arms demonstrate is the results of their unique musculature, which allows them to function without a distinct skeletal support system. Behavioral studies of these creatures reveal the unique way in which they utilize their musculature to perform a wide variety of tasks.

\section{A. Octopus Arm Morphology (Muscular Hydrostats)}

Biological skeletal support systems fall into two general categories. One category features skeletal systems composed of hardened elements such as the bones of vertebrate animals or the exoskeleton of arthropods. The hardened elements in a traditional hard skeleton provide attachment points for muscle and constrain muscle motion in a desired direction. These skeletal supports also provide lever and pivot points that amplify the resultant force, speed, or displacement of muscle activity and make certain movements possible.

The second skeletal support system category features hydrostatic skeletons. This kind of system can be found in polyps and vermiform animals (e.g. sea anemone, earthworm). Hydrostatic skeletons rely on flexible fluid-filled cavities that are constrained by connective tissue to conform to a given shape, typically cylindrical. These skeletons utilize the constant volume principle to produce movement. When the radial muscles situated around the cavity contract, they reduce the diameter of the cavity which forces the cavity to elongate to maintain constant volume. Similarly when the longitudinal muscles contract, they reduce the length of the cavity which causes the cavity to increase in diameter.

In most cases, these skeletal support systems are the means by which forces produced by muscle contraction are transmitted to produce movement. However, there are a number of biological structures that are almost entirely composed of muscle that operate without distinct skeletal support. Tongues, elephant trunks, and octopus arms are examples of such structures, dubbed muscular hydrostats in [18]. The arrangement of the musculature in these structures tends to be densely packed with fibers oriented in three general directions: (1) perpendicular to the long axis, (2) paral- 
lel to the long axis, and (3) oblique around the long axis [18].

For the octopus arm, the perpendicular muscle fibers are packed in a transverse arrangement, alternating horizontally and vertically. The parallel muscle fibers are set in longitudinal bunches around the center of the arm. These bunches are grouped antagonistically along the top and bottom of the arm and the left and right side of the arm. Additionally, the oblique muscles are arranged in antagonistic bunches (right-handed and left-handed helixes) along the periphery of the arm.

Muscular hydrostats rely on the constant-volume principle to produce motion, in a fashion similar to traditional hydrostats. However, unlike traditional hydrostats, there is no distinct fluid-filled cavity in muscular hydrostats. Instead, the hydrostatic fluid is contained within the muscle cells, which means that muscular hydrostats could in effect be modeled as hyperredundant traditional hydrostats. More chambers of hydrostatic fluid gives muscular hydrostats appreciably more localized movement control than traditional hydrostats have.

Unilateral elongation can be performed by contracting the transverse muscles while relaxing the longitudinal muscles. This muscle actuation configuration reduces the cross-sectional area of the arm causing a corresponding increase in length. The alternating horizontal and vertical arrangement of the transverse muscles adds a degree of control, allowing the arm to flatten itself in a particular direction. A radial arrangement of the muscles would allow only for a general decrease in the cross-sectional area, as is the case with the traditional hydrostats. Unilateral shortening can similarly be performed by contracting the longitudinal muscles while relaxing the transverse muscles. This muscle actuation configuration reduces the length of the arm causing the cross-sectional area to increase in response.

More complex arm bending and stiffening actions can be produced by combining activity of the transverse and longitudinal muscles. One way that the arm can be bent is by contracting the longitudinal muscles on one side of the arm while also contracting the transverse muscle. Contracting the transverse muscles causes the arm to resist the increase in diameter (and corresponding decrease in length) that contraction of the longitudinal muscles would otherwise cause. This resistance provides the structural stability required for bending. The degree and strength of the bend can be modulated by the level of contraction of both the transverse and the longitudinal muscles. Stiffness without motion can be accomplished by contracting the transverse and the longitudinal muscles so that the forces they exert on the shape of the arm balance out. Thus, while the arm is in a static configuration, the tension in the muscles will make the arm stiffer and more resistant to external forces.

The oblique muscles can be used to twist the arm along its long axis. The direction of this torsion depends on how the oblique muscle is helically arranged. Contraction of the muscle that is arranged as a righthanded helix causes counter-clockwise torsion in the arm; whereas, the opposite is true for contraction of the left-handed helix muscle. These oblique muscles may also play a part in elongation and stiffening of the arm.

Localized motion is the result of localized control of the muscles in the octopus arm. Varying the pattern and coordination of the muscular activity in the arm allow the specific direction, degree, strength, and point of bend to be freely modified. Additionally, localized extension/retraction, torsion, and stiffening allow the octopus to produce an impressive array of motions and behaviors with its arm. In this way, the muscularhydrostat system is more flexible and adaptable than most other musculoskeletal systems.

Performing such complex local muscle control requires considerable neural capacity. The nervous system of an octopus is quite diffuse, with most of its neurons being found outside of the brain. More than twice as many nerve cells are found in the arm nerve cords than in the brain, which suggests that much of the control processing for octopus arms is distributed and lies within the arms [19, p. 246-7].

Feedback for control must be guided by a sensory system. In octopus arms, much of the tactile sensory system lies in the suckers. Further analysis of the morphology of the suckers on octopus arms (and synthesis of artificial suckers) is currently underway at Brooklyn College ${ }^{1}$.

To demonstrate that high-level behaviors are made possible by this unique muscular structure, models based on the musculature of the octopus have been formulated to artificially simulate these behaviors. These models can also be used to aid in the design of a robotic manipulator with similar properties. More information about the morphology of cephalopod limbs can be found in $[18,19]$.

\footnotetext{
${ }^{1}$ More information on this research can be found at http://academic.brooklyn.cuny.edu/userhome/psych/fgrasso/.
} 


\section{B. Octopus Arm Behavior}

Octopi demonstrate an impressive variety of behaviors using their arms. The arms can perform a variety of reaches, pulls, sweeps, arm tip wraps, rotational twists and grasps. These basic behaviors can be combined to perform more complex behaviors, such as locomotion and feeding. While octopi often use their mantle cavity to propel themselves through the water, they will also use their arms to "walk." The octopus performs a walking motion by reaching its arms out, grasping points in the direction of motion, and pulling itself toward those points. Grasping is accomplished either by adhering with its suckers to a surface, wrapping its arm around a stable object, or a combination of these two actions.

Octopi have several feeding behaviors, but perhaps the most interesting with respect to robotic manipulators is what happens when an octopus feeds on a shrimp. To capture a shrimp, the octopus dynamically reaches its arms out with a rolling action toward the shrimp. Then, the portions of the arms that come into contact with the shrimp immediately wrap tightly around the shrimp to ensure a stable grasp. An "elbow" or bending point is formed along the arm about halfway between the location of the shrimp on the arm and the location of the octopus mouth, enabling the octopus to easily put the shrimp in its mouth [20]. The flexibility of choosing a bending point anywhere along the arm is an ability unique to muscular hydrostats. Such an ability, if duplicated, would create a highly adaptable robotic manipulator. Some initial investigations into continuous backbone robots with variable bending locations are given in [21].

Observation of the arm behavior of the octopus reveals that the purpose of all arm movement seems to be to bring the sucker surface to bear on a task. The suckers provide not only adhesion, but also contain a wide variety of tactile and chemosensory receptors. The suckers are also remarkably dexterous and are even used to independently locomote the octopus under some conditions. An object can also be moved along the octopus arm by being passed from sucker to sucker. This knowledge of octopus behavior leads towards consideration of a manipulator designed with a single preferred grasping side.

As discussed in the previous section, the arms contain significant neural mass. This enables them to perform reflex actions, such as bringing food to the creature's mouth without any input from the brain. Also, much of the proprioception (self-sensing) system required for manipulation seem to be within the neural system in the arms, and not relayed back to the brain [20]. For instance, octopus seem unable to perceive weight differentiation among objects they manipulate [19, p. 233-237]. When they are given cylinders of differing weight, the arm obviously compensates for heavier cylinders with increased muscle tension, however researchers are unable to train them to choose a heavy cylinder over a light cylinder or vice versa.

These observations suggest the presence of a distributed control system in the form of low-level motor programs which reside in the arms neural mass. Presumably the brain of the octopus need only send (relatively) higher level signals to the arm, and the neural system within the arm breaks it down into lower level actions. This relationship between the octopus arm and brain provides some insight as to how an octopus-arm robotic manipulator might be remotely controlled by a human operator.

\section{Octopus Arm Modeling}

Forming a model of how the octopus' arms convert muscle contractions to useful behavior provides the following. First, it helps validate the theory of how muscular hydrostats are capable of movement without a distinct skeletal support structure. Second, it provides further insight into how the control systems of the animals utilize the muscles to perform actions. Third, a model connects biology to engineering, allowing the development of a robotic manipulator which observes key aspects of the biological model.

Due to the complexity of the octopus arm, a number of simplified modeling approaches have been proposed which together provide a series of tradeoffs in accuracy and complexity. Complex, fully soft models attempt to describe the action of each muscle fiber as it interacts with others in an arm. The cylindrical extension model assumes an arm can be divided into a series cylinders varying in radius connected end to end. This model clearly illustrates the mechanical principles behind the operation of a muscular hydrostatic skeleton. Models based on a flexible backbone provide octopus arm dynamics, like soft models, but assume an extension-less system to simplify the model's complexity. Finally, circular curvature models provide a very simple description of an octopus arm while making somewhat restrictive assumptions about the arm's shape.

Each proposed model provides insight into the biomechanics of the octopus' arm and suggests an accompanying implementation for a robotic manipulator. The 
following paragraphs examine some of the proposed models.

\section{A. Soft model}

One method for modeling muscular hydrostats is to model the arm as a collection of constant-volume cylinders. Each cylinder can exert force radially, changing its diameter, or axially, changing its length, in much the same way the transverse and longitudinal muscles in an octopus arm act. The forces generated by each cylinder can be derived from measurements of the muscle properties of squid [22]. These cylinders are grouped and surrounded by a "skin" of connective tissue to form an arm.

A second approach is to model the arm as a set of cubes connected end to end, each of which can be bent in two directions, again modeling the longitudinal and transverse muscles in an octopus arm. Simulations with this model reveal that a simple sequential contraction of transverse muscles in a bent arm produces the characteristic arm roll that octopus often use when reaching [20].

However, the complexity of these models introduces two challenges. First is the ability to regulate the position of the arm to a desired position. Second is the difficulty in developing a mechanical system composed entirely of soft, constant-volume actuators. Neither of these challenges have as of yet been solved.

\section{B. Cylindrical model}

An alternative is to model the arm as a series of constant-volume cylinders joined end-to-end [22]. This model provides insight into how muscular hydrostats convert small displacement, high force muscle tissue contractions into high displacement, low force arm movements. This forward dynamics model, using constants obtained from measurements of squid musculature, accurately predicts the motion of a squid's tentacle during a prey strike. While this model provides excellent insight into the behavior of the muscular hydrostat, it essentially describes a single prismatic joint in robotic terms. Therefore, it does not describe the full function of a useful robot.

\section{C.Flexible backbone}

Another approach is to model the arm as a constantstiffness rod, which bends due to discrete or continuous forces applied to it. With sufficient simplifying assumptions, a dynamic model can be developed [23]. One of the most limiting of these simplifying assumptions, with respect to octopus arm behavior, is the assumption that the constant-stiffness rod is incapable of

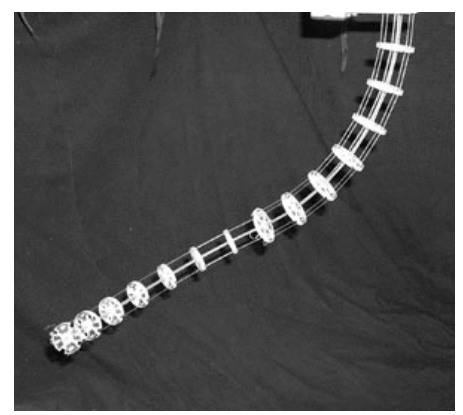

Figure 1: Tentacle Manipulator

extension, retraction, or torsion. Another limit of this model is that the dynamic equations have only been solved for the two-dimensional case; extension to three-dimensions has proven to be extremely challenging.

This model provides a reasonable, if limited, model for an octopus arm and also a model for constructing a robot based on a flexible rod. The Tentacle Manipulator (shown in Figure 1) was constructed at Clemson University following this model [23]. The core of the manipulator is a thin continuous elastic rod, while pairs of remotely actuated tendon-cables simulate muscle. Unfortunately, the restriction of the model to twodimensions limits its practical application.

\section{Circular assumption}

The model that currently has the most practical application is based on the assumptions that the arm bends in sections with constant curvature and is incapable of torsion. Using these assumptions, the system can be simply modeled as series of revolute and prismatic joints, which allows the forward kinematics to be defined using the traditional Denavit-Hartenberg technique. This is advantageous because it facilitates the use of traditional robot control algorithms.

The novel Elephant Trunk robotic manipulator (shown in Figure 2) at Clemson University was controlled based on this model. The trunk is comprised of 32 degrees of freedom in 16 small links. There are four sections with two controllable degrees of freedom each. The sections are actuated with two pairs of antagonistically arrayed cable-tendons. The kinematics for the trunk are derived in [4].

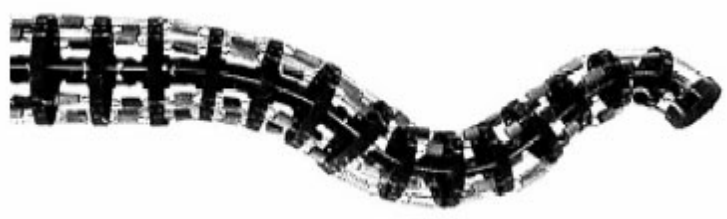

Figure 2: Elephant Trunk manipulator 
Recently, the novel continuum robot Air-Octor (shown in Figure 3) was also designed based on this model. This design is novel in that it provides a combination of pneumatic and tendon actuation for controlled bending and extension as well as a double skin concept which provides a smooth continuum exterior for environmental interaction. The tendon configuration in this manipulator has also been modified so that there are three cables per section, rather than four as in the Elephant Trunk. To control this manipulator the kinematics had to be extended to handle extension and retraction and adapted to deal with a three tendon configuration [24]. The hardware realization and the kinematic derivation are discussed in more detail in the following sections.

\section{Air-Octor Physical Description}

The prominent design feature of the Air-Octor manipulator is a pneumatically pressurized central chamber sealed by aluminum end caps. This pressurized chamber acts as a continuously deformable backbone that provides the structural rigidity necessary to make the manipulator useful. Essentially, this central chamber is analogous to the transverse muscles in the octopus arm. One of the advantages of this design is that it frees up a large amount of space in the center of the manipulator that would otherwise be made up of a solid backbone structure. The space within the central chamber provides room for the routing of wires/hoses that may be needed to accommodate sensors or payloads.

An outer tube is fitted over the central chamber to provide protection for the central hose and a smooth durable surface with which to perform whole-arm manipulation. Protection is needed as holes or tears in the

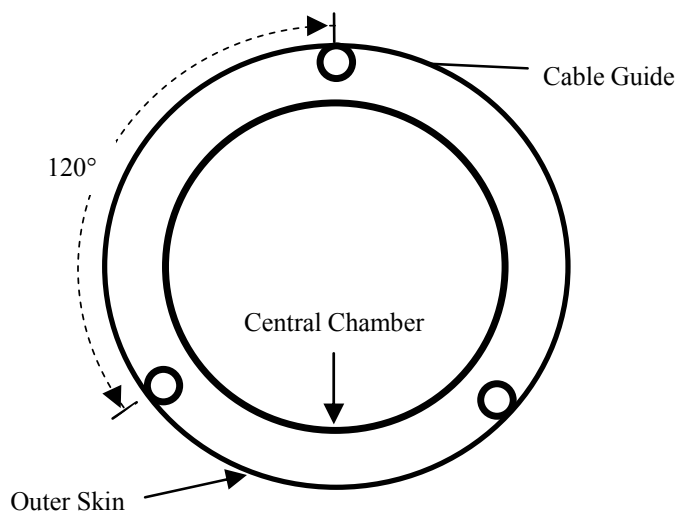

Figure 4: Cross-sectional schematic of the skin and tendon configuration.

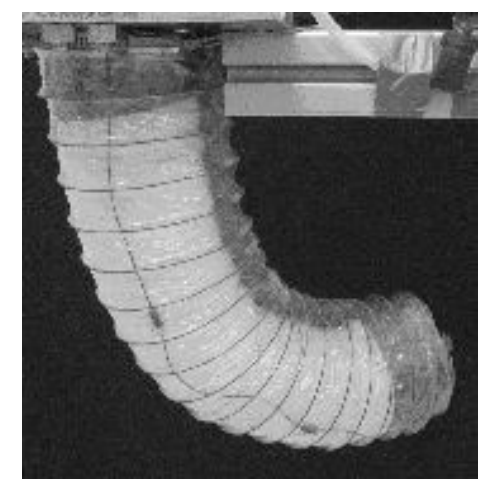

Figure 3: Air-Octor single manipulator section in a bent configuration.

central hose would destroy its effectiveness as a flexible backbone. Cable guides are attached to the interior of the outer tube to route three individual tendon cables straight along the length of the manipulator. The cables are then anchored to the aluminum end cap at the end of each manipulator section. These cables are analogous to the longitudinal muscles in the octopus arm.

The primary motion of the manipulator is controlled by a tendon (cable) servo system. Standard DC motors actuate the tendons via a spooling system. The three tendons are arranged antagonistically about the manipulator section in a 120-degree configuration as shown in Figure 4. This antagonistic arrangement is necessary because the cables can only exert a pulling and not a pushing force. A pulling force on one of the cables causes the manipulator section to bend in the direction of the pulling cable. The combination of the three cables allows the manipulator section to be bent in any direction. The cable configuration used gives the manipulator section two degrees of freedom in bending.

The "soft construction" of the manipulator provides a third degree of freedom. Through appropriate actuation of the three tendon-cable motors, the manipulator can be made to retract and extend. The total length of the manipulator when fully retracted is approximately $10 \mathrm{~cm}$, which gives the manipulator a 5:1 retraction ratio. This extension/retraction motion is improved with the addition of an electro-pneumatic servo pressure regulator to the system. The addition of the servo pressure valve allows for control of the overall compliance of the manipulator by varying the pressure of the central chamber. This is analogous to stiffness modulation via muscle contraction in the octopus. Further detail can be found in [25]. 


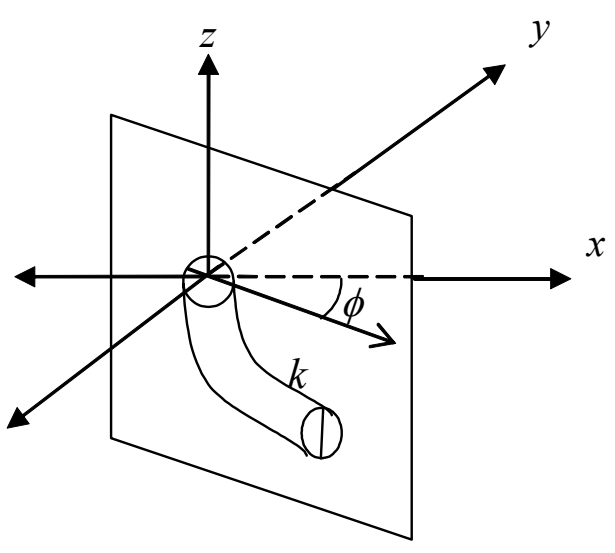

Figure 5: Manipulator variables $\phi$ and $k$. The variable $\phi$ gives the rotation in the $x y$ plane.

\section{Air-Octor Kinematics}

Given the novelty of the Air-Octor, the task of developing kinematic models (relating robot shape to input cable lengths) has proved to be a significant challenge. The following section describes the derivation of the corresponding inverse kinematic model (finding cable lengths given shape). The forward kinematics model (finding shape given cable lengths) is also mentioned in the next section, but in the interest of space is the derivation is not discussed. The detailed derivation of the forward kinematics model can be found in [24] and [26].

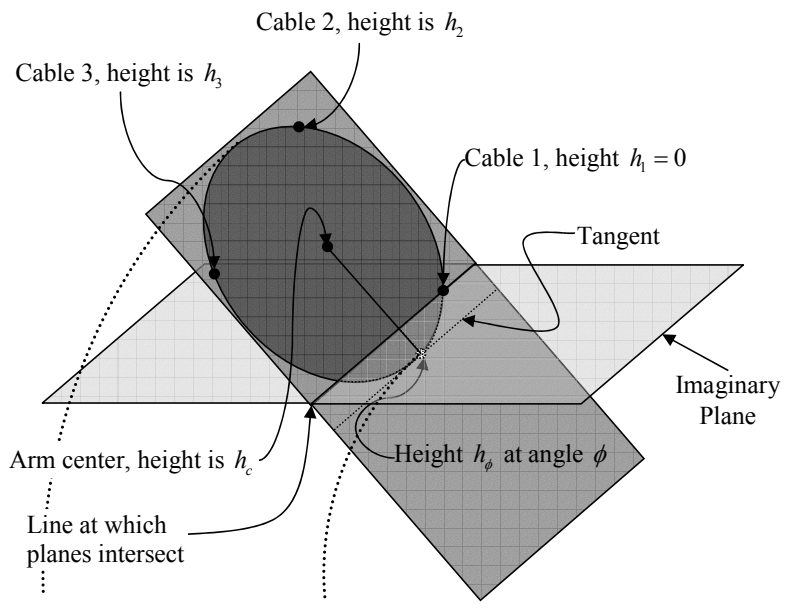

Figure 6: Intersection of plane containing manipulator top and imaginary reference plane

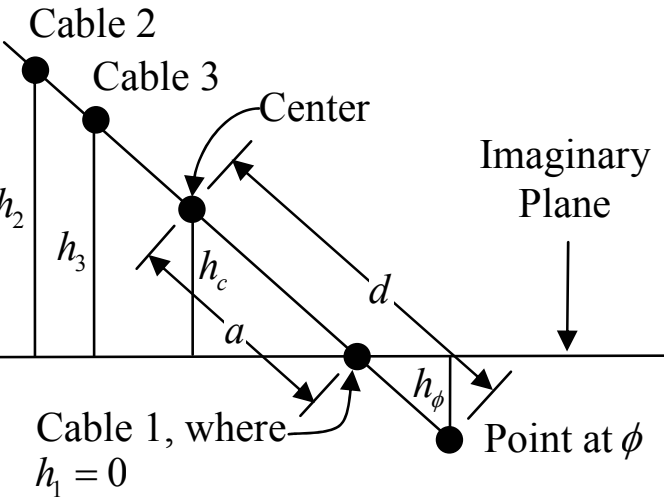

Figure 7: End-on view of planar intersection shown in Figure 6

\section{A.Transformation from manipulator curvature to cable lengths}

Consider the manipulator section show in Figure 5. Given the length of one section of the manipulator s, the curvature of that section $k$, the angle at which the curvature lies $\phi$, the distance from the center of the manipulator to the location of the cables $d$, and the number of segments in this manipulator section $n$, this section discusses computing the lengths of the three cables $l_{1}, l_{2}$, and $l_{3}$ due to the configuration of the manipulator section. This is the inverse kinematics problem.

The top of the manipulator can be viewed as lying in a plane with the $(\mathrm{x}, \mathrm{y})$ plane. As shown in Figure 6, this plane can be shown to intersect with an imaginary reference plane to form a line. The line of intersection lies parallel to a tangent line that runs through the point specified at angle $\phi$. Viewing the intersection of the

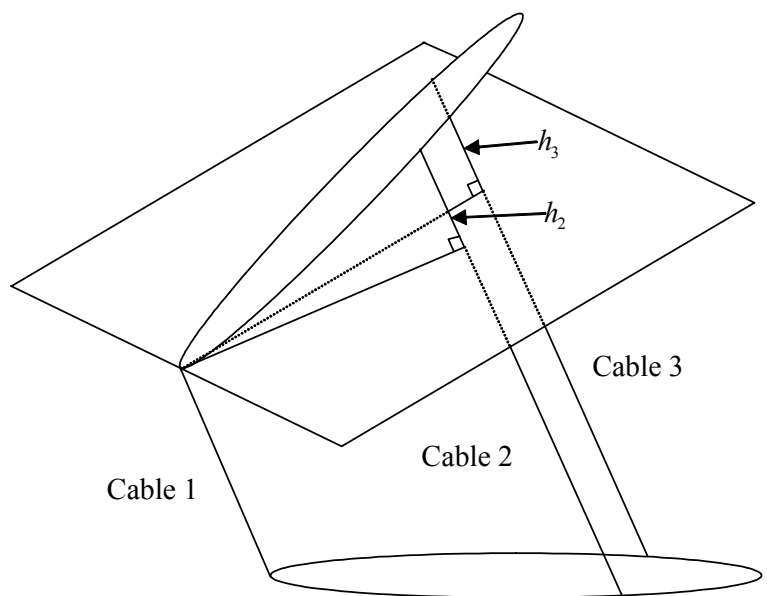

Figure 8: Bent manipulator section with imaginary reference plane. 


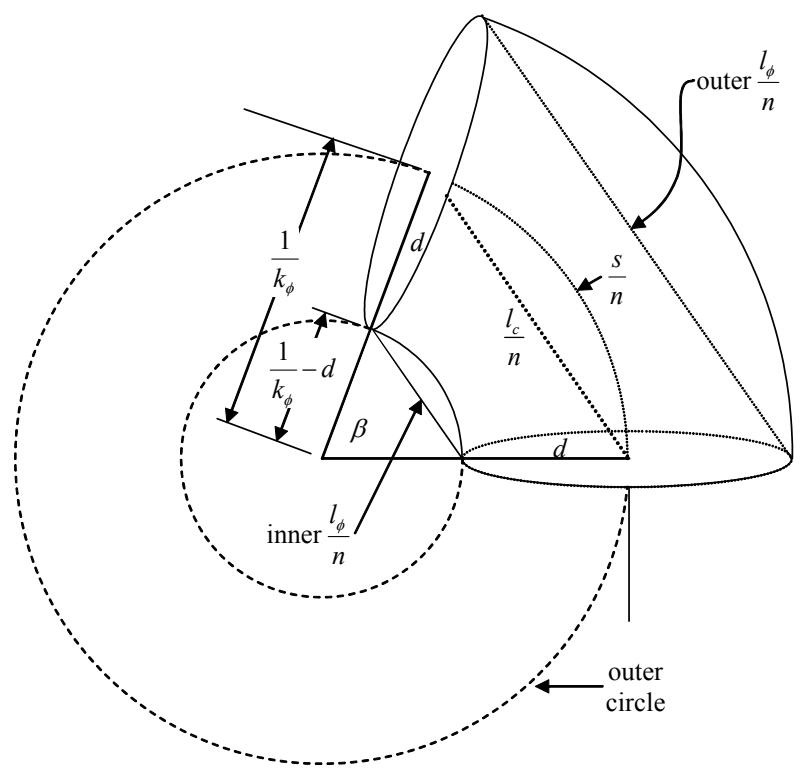

Figure 9: Geometry of bent manipulator section

two planes end-on, as in Figure 7, shows that any position on the plane with the circle can be projected along a line parallel to the tangent line onto the diagonal line. Knowing the slope and a position on this line allows computation of the height of the position. The height of a position is defined as the distance of that position from the imaginary plane. (The imaginary plane is depicted as the horizontal line in Figure 7.) Therefore, the height of any point on the circle, such as $h_{c}$, can be determined by projecting the point onto the diagonal line and then applying the slope-intercept form of that line to find the point's height. In the forward kinematics derivation [26], the height $h_{1}=0$ was chosen to anchor the plane as shown in Figure 6, Figure 7, and Figure 8 . For the inverse kinematics derivation, the

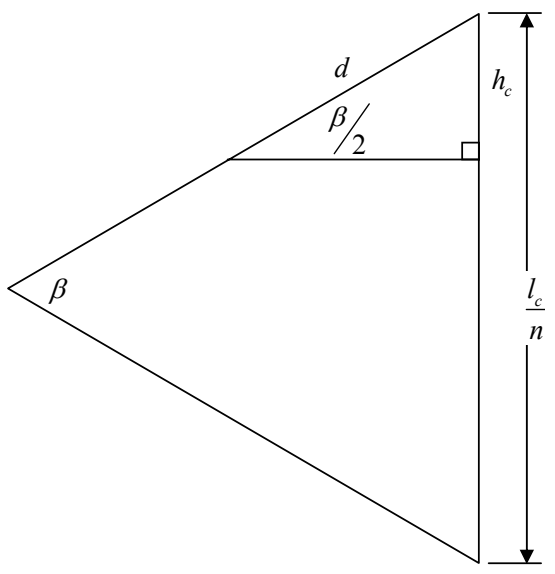

Figure 10: Triangle from Figure 9 used to relate $h_{c}$ to $\beta$. plane will be anchored by $h_{\phi}=0$ instead.

Redrawing the triangle with sides $\frac{1}{k_{\phi}}$ and $\frac{l_{c}}{n}$, shown in Figure 9, creates Figure 10 which reveals $\sin \left(\frac{\beta}{2}\right)=\frac{h_{c}}{d}$. In a circle of radius $r$, the angle $\varphi$ subtended by a portion of the circumference of length $a$ obeys the following relationship, $\frac{2 \pi r}{a}=\frac{2 \pi}{\varphi}$. To compute $\beta$ for the outer circle shown in Figure 9, substitute $r=\frac{1}{k_{\phi}}, a=\frac{s}{n}$, and $\varphi=\beta$ into the above equation and solve for $\beta$ to get $\beta=\frac{s k_{\phi}}{n}$. Substitute this value for $\beta$ into the previous equation and solve for $h_{c}$ to get $h_{c}=d \sin \left(\frac{s k_{\phi}}{2 n}\right)$.

Projecting these points onto the diagonal line of Figure 7 allows the line to be defined as $h_{1 p}=h_{1}=\frac{h_{c}-h_{\phi}}{d}(d-a)+h_{\phi}$. As illustrated in Figure 11 , projecting the height $h_{1}$ along a line parallel to the tangent gives $h_{1}=h_{1 p}$, where $h_{1 p}$ is the height at a point along the line connecting $h_{c}$ and $h_{\phi}$. Also note from Figure 11, that $\cos \left(\frac{\pi}{2}-\phi\right)=\frac{a}{d}=\sin \phi$. Substitute and simplify to get $h_{1}=d \sin \left(\frac{s k_{\phi}}{2 n}\right)(1-\sin \phi)$.

Following the reasoning in [26], the length of cable $1 l_{1}$, can be divided into $l_{\phi}$, the length of a virtual cable

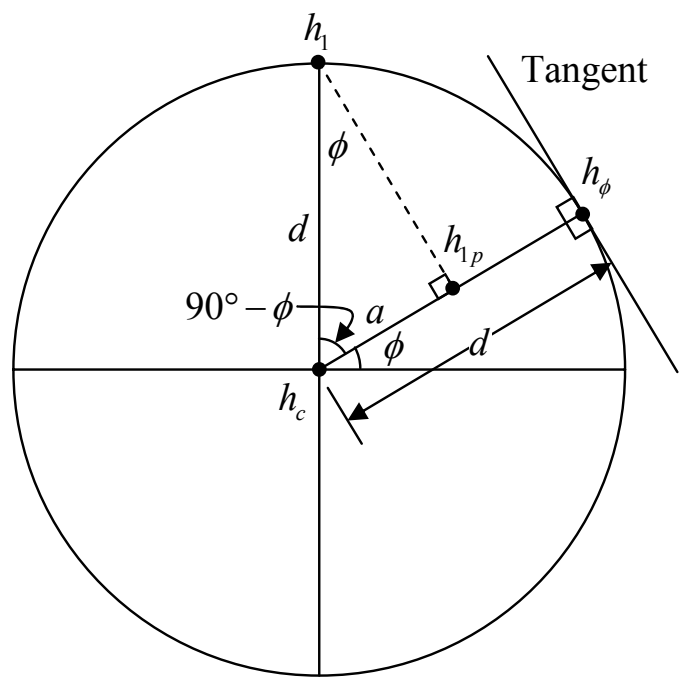

Figure 11: Projecting $h_{1}$ along a line parallel to the tangent (as in Figure 7). 


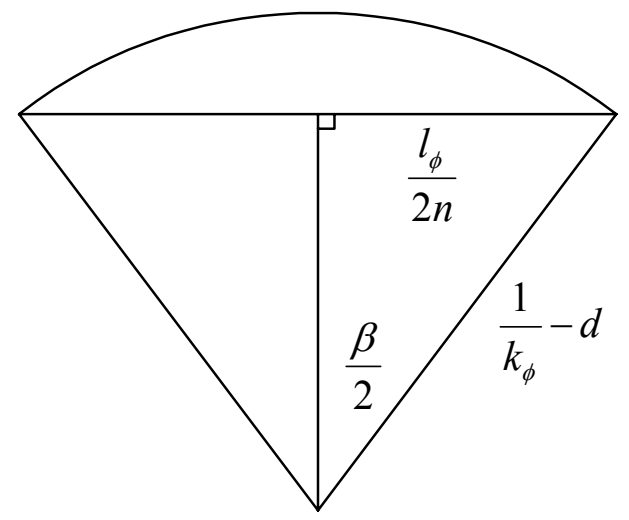

Figure 12: Triangle used to determining cable length $l_{\phi}$. (from Figure 9).

attached at angle $\phi$ on the circumference of the manipulator and running the length of the manipulator, and $h_{1}$, the height cable 1 extends above the plane anchored by $l_{\phi}$, so that $l_{1}=l_{\phi}+2 n h_{1}$. To find $l_{\phi}$, note that the length of a cable $l_{\phi}$ attached to the circumference of the manipulator at the point of maximum curvature $\phi$ forms the triangle shown in Figure 12, yielding $\sin \left(\frac{\beta}{2}\right)=\frac{\frac{l_{\phi}}{2 n}}{\frac{1}{k_{\phi}}-d}$. Therefore, the cable length can be computed as $l_{\phi}=2 n\left(\frac{1}{k_{\phi}}-d\right) \sin \left(\frac{s k_{\phi}}{2 n}\right)$. Substituting and simplifying,

$l_{1}=2 n \sin \left(\frac{s k_{\phi}}{2 n}\right)\left(\frac{1}{k_{\phi}}-d \sin \phi\right)$

Likewise, rotating $\phi$ to $210^{\circ}$ and $330^{\circ}$, the locations of the attachment points of cables 2 and 3, then resolving produces

$$
\begin{aligned}
& l_{2}=2 n \sin \left(\frac{s k_{\phi}}{2 n}\right)\left(\frac{1}{k_{\phi}}+d \sin \left(\frac{\pi}{3}+\phi\right)\right) \text { and } \\
& l_{3}=2 n \sin \left(\frac{s k_{\phi}}{2 n}\right)\left(\frac{1}{k_{\phi}}-d \cos \left(\frac{\pi}{6}+\phi\right)\right) .
\end{aligned}
$$

Note that because $\lim _{x \rightarrow 0} \sin x=x$, in the limiting case $\lim _{k_{\phi} \rightarrow 0} l_{1}=2 n\left(\frac{s k_{\phi}}{2 n}\right)\left(\frac{1}{k_{\phi}}-d \sin \phi\right)=s$. In the same way, $\lim _{k_{\phi} \rightarrow 0} l_{2}=\lim _{k_{\phi} \rightarrow 0} l_{3}=s$.

\section{B. Transformation from cable lengths to manipulator curvature}

As discussed in [26], the forward kinematics for the manipulator can also be derived using geometrical methods. Defining $g \triangleq l_{1}^{2}+l_{2}^{2}+l_{3}^{2}-l_{1} l_{2}-l_{2} l_{3}-l_{1} l_{3}$, this analysis yields

$$
\begin{aligned}
& k_{\phi}=2 \frac{\sqrt{g}}{d\left(l_{1}+l_{2}+l_{3}\right)} \\
& \phi=\tan ^{-1}\left(\frac{\sqrt{3}}{3} \frac{l_{3}+l_{2}-2 l_{1}}{l_{2}-l_{3}}\right) \\
& s=\frac{n d\left(l_{1}+l_{2}+l_{3}\right)}{\sqrt{g}} \sin ^{-1}\left(\frac{\sqrt{g}}{3 n d}\right)
\end{aligned}
$$

where $\lim _{g \rightarrow 0} s=\frac{l_{1}+l_{2}+l_{3}}{3}$.

The above kinematic models have been implemented in real time on Air-Octor. Thus far, they have been found to be effective in predicting both the shape of the arm for given tendon lengths (forward kinematics) and providing the low-level tendon feedback control system with effective nominal trajectories required to drive the robot to a desired shape (inverse kinematics). These implementation results will be reported in more detail in future publications. Further details on the kinematic derivations can be found in [24].

\section{Results and Conclusions}

This paper discusses ongoing research into the arms of octopus, focusing on how models developed to understand and explain their operation can be used in the design and operation of octopus-like robot arms. By extracting general principles of motion from octopus arms, such as using longitudinal muscles to generate bending by pulling against variable stiffness, a model for a simple but effective robot arm was generated, and appropriate kinematics derived. A novel manipulator design that uses this model has been implemented in hardware and appropriate kinematic models have been developed to control the hardware.

In future hardware work, additional degrees of freedom will be added to the manipulator by attaching additional sets of tendon cables. Terminating a second set of tendons at some point along the existing manipulator, except at the tip, provides an additional two degrees of controllable bending freedom and an additional degree of extension/retraction freedom. The number of degrees of freedom also can be increased by mounting a second segment on the manipulator, thus directly creating a two-section manipulator.

Additional work is being performed to derive the proper velocity kinematics that will allow the manipulator to be remotely operated with a joystick. Also, 
whole arm manipulation algorithms using the current kinematics models are being developed.

\section{References}

[1] G. Robinson and J. B. C. Davies, "Continuum Robots A State of the Art", In: Proceedings 1999 IEEE International Conference on Robotics \& Automation, pp. 2849 2854.

[2] H. Takanobu, T. Tandai, and H. Miura, "Multi-DOF Flexible Robot Based on Tongue", In: Proceedings 2004 IEEE International Conference on Robotics \& Automation, pp. 2673-8.

[3] R. Cieslak and A. Morecki, "Elephant Trunk Type Elastic Manipulator - A Tool for Bulk and Liquid Type Materials Transportation", In: Robotica, Vol. 17, 1999, pp. 11-16.

[4] M.W. Hannan and I.D. Walker, "Kinematics and the Implementation of an Elephant's Trunk Manipulator and Other Continuum Style Robots", In: Journal of Robotic Systems, 20(2), 2003, pp. 45-63.

[5] K. Suzumori, S. Iikura, H. Tanaka, "Development of Flexible Microactuator and its application to Robotic Mechanisms", In: Proceedings 1991 IEEE International Conference on Robotics \& Automation, pp. 1622-7.

[6] H. Tsukagoshi, A. Kitagawa, and M. Segawa, "Active Hose: an Artificial Elephant's Nose with Maneuverability for Rescue Operation", In: Proceedings 2001 IEEE International Conference on Robotics \& Automation, pp. 2454-2459.

[7] J.F. Wilson, D. Li, Z. Chen, and R.T. George Jr, "Flexible Robot Manipulators and Grippers: Relatives of Elephant Trunks and Squid Tentacles", In: Robots and Biological Systems: Toward a New Bionics?, 1993, pp.4749.

[8] R. Buckingham and A. Graham, "Reaching the unreachable - snake-arm robots", Presented at International Symposium of Robotics, 2003, Available via: OCRobotics Ltd. http://www.ocrobotics.com.

[9] I.A. Gravagne and I.D. Walker, "On the Kinematics of Remotely-Actuated Continuum Robots", In: Proceedings 2000 IEEE International Conference on Robotics \& Automation, pp. 2544-50.

[10] G. Immega and K. Antonelli, "The KSI Tentacle Manipulator", In: Proceedings 1995 IEEE Conference on Robotics \& Automation, pp. 3149-54.

[11] H. Ohno and S. Hirose, "Design of Slim Slime Robot and its Gait of Locomotion", In: Proceedings 2001 IEEE/RSJ International Conference on Intelligent Systems, pp. 707-15.

[12] Hallam, J. "Can we mix robotics and biology?", In: Proceedings 1998 IROS Workshop WT1: Defining the Future of Biomorphic Robot.

[13] S. Vogel, Cats' Paws and Catapults, Norton \& Co, New York, 1998.
[14] G.S. Chirikjian, "Theory and Applications of Hyperredundant Robotic Mechanisms", Ph.D. thesis, Department of Applied Mechanics California Institute of Technology, 1992.

[15] I.A. Gravagne. "Design, Analysis and Experimentation: The Fundamentals of Continuum Robotic Manipulators", Ph.D. Dissertation, Department of Electrical and Computer Engineering, Clemson University, August 2002.

[16] S. Hirose. Biologically Inspired Robots. Oxford University Press. 1993.

[17] H. Mochiyama, E. Shimemura, and H. Kobayashi, "Direct Kinematics of Manipulators with Hyper Degrees of Freedom and Frenet-Serret Formula", In: Proceedings 1998 IEEE International Conference on Robotics and Automation, pp. 1653-58.

[18] W. Kier and K. Smith, "Tongues, tentacles, and trunks: the biomechanics of movement in muscular hydrostats", Zoological Journal of the Linnean Society, vol. 83, 1985, pp. 307-324.

[19] M.J. Wells, Octopus: Physiology and Behavior of and Advanced Invertebrate, John Wiley \& Sons, New York, 1978.

[20] G. Sumbre, T. Flash, G. Fiorito, and B. Hochner, "Motor control of the octopus' fetch movement". Annual meeting of the Israeli Society for Neuroscience, Eilat, Israel 14-16 Dec. 2003.

[21] M. Blessing and I.D. Walker, "Novel Continuum Robots with Variable-Length Sections", to appear, 3rd IFAC Symposium on Mechatronic Systems, Sydney, Australia, September 2004.

[22] J. L. van Leeuwen and William M. Kier, "Functional Design of Tentacles in Squid: Linking Sarcomere Ultrastructure to Gross Morphological Dynamics", Philosophical Transactions: Biological Sciences, vol. 352, May 29, 1997, pp. 551-571.

[23] I.A. Gravagne, I.D. Walker, and C.D. Rahn, "Large Deflection Dynamics and Control for Planar Continuum Robots", IEEE/ASME Transactions on Mechatronics, Vol.8, No.2, June 2003, pp 299-307.

[24] B. A. Jones. "Kinematics of a Novel Pneumatic Manipulator." Technical report, Dept. of ECE, Clemson University, February 2004.

[25] W. McMahan. "Pneumatic Hose Tentacle Manipulator (Air-Octor)", Technical report, Dept. of ECE, Clemson University, April 2004.

[26] B. Jones, W. McMahan, and I.D. Walker, "Design and Analysis of a Novel Pneumatic Manipulator", to appear, 3rd IFAC Symposium on Mechatronic Systems, Sydney, Australia, September 2004. 